\section{Optimal Parameters for DEM Extraction from IKONOS data: A case Study of a Coastal Area Mariluz Gil-Docampo* and Marcos Arza-García}

\begin{abstract}
The gap between aerial and satellite photogrammetry is getting smaller as both systems have benefited from great technology improvements in the last years. However, the major advantage of earth observation satellites continues to be the possibility to acquire data virtually anywhere on the planet without considering border and logistical restrictions and over huge extensions. A pair of stereo images corresponding to a coastal area in the NW of Spain is the basis for this paper, which analyzes certain influential variables in the process of generating a Digital Elevation Model (DEM) from stereoscopic pairs of IKONOS images. The mathematical model, the number of Ground Control Points (GCPs) and their accuracies are analyzed through a sequence of experimental trials. Two main methods are applied for math modeling: a CCRS model (rigorous model) and a Rational Functions model. The influence of the GCPs' positional quality is compared using two different information sources: points measured in 1:5,000 cartography and points surveyed via GPS. The number of GCPs tested varies between 0 and 20. Using different configurations of these variables, 17 models are generated. The best results are achieved with the rigorous model and $16 \mathrm{GCPs}$ measured with GPS with an RMSE of $1.01 \mathrm{~m}$ (or an LE95 -Linear Error in Z at $95 \%$ confidence level- of $2 \mathrm{~m}$ ), which is approximately the pixel size of the initial pair. The mathematical model was determined to be the variable with the most influence on accuracy. Moreover, the results suggest that the use of 10-16 GCPs is sufficient. Additional points do not improve the DEM accuracy or may even worsen it in certain cases. Although the study is carried out in a coastal zone, which restricts the GCPs distribution possibilities, the results are comparable with similar results from inland areas.
\end{abstract}

\section{Keywords}

Satellite photogrammetry; Mathematical model; Rigorous model; Rational functions; GCPs; Vertical accuracy; LE95

\section{Introduction}

\section{DEM generation in large areas}

Direct methods for DEM generation, such as a topographic survey using total station or GPS (Global Positional System), are considered techniques with high accuracy, but they require a large investment in terms of economics and time. For this reason, the use of these approaches is limited to large-scale cartographic surveys or small

*Corresponding author: Mariluz Gil-Docampo, Agroforestry Engineering Department, Higher Polytechnic School, 27002 Lugo, Spain, Tel: 0034647344115 E-mail: ml.gil@usc.es

Received: September 29, 2017 Accepted: October 16, 2017 Published: October 24, 2017 areas (civil engineering works or other projects located in specific areas). For modeling in larger areas, other techniques are available, including photogrammetry or LIDAR (Light Detection and Ranging) [1] or techniques that use spatial sensors, such as radargrammetry [2] or IFSAR (Interferometric Synthetic Aperture Radar) [3], or the use of stereo images from optical sensors.

The advent of Very High Resolution (VHR) optical satellites capable of producing stereo images led to a new era in extracting DEMs which commenced with the launch of IKONOS. Although high-resolution satellites cannot provide a substitute for aerial photogrammetry in terms of resolution, their major advantage continues to be the possibility to acquire data virtually anywhere on the planet without considering border and logistical restrictions and over huge extensions.

\section{IKONOS stereo imagery}

The investigations carried out from IKONOS stereo images are aimed at different objectives, and the working methodologies are also accordingly rather different. Therefore, the results are obtained with reliability and accuracy levels that also differ significantly.

Many studies are not directly focused on evaluation of the positional quality but rather on the potential applications of the images depending on their positional quality; this perspective is exemplified in the work carried out [4], which analyzes the feasibility of the use of stereoscopic pairs of IKONOS images for the three-dimensional reconstruction of buildings; the work [5], which proposes the combined use of DEMs and the spectral information from IKONOS to perform 3-D thematic models (i.e., a model of mulches); and [6] which studies the 3-D modeling of urban areas from a stereoscopic pair.

In addition to 3-D city modeling, many other applications exist that present the use of DEMs derived from IKONOS images. A practical methodology for coastal line mapping is presented in Kaichang et al. [7]. On the other hand, Shaker et al. [8] carried out an analysis of the possibilities of an IKONOS stereoscopic pair for mapping of existing structures, focusing mainly on highways, and analyzed the results using the source of greatest accuracy as a reference: that obtained from 1:1,000 topographic mapping of the same area. The results show a discrepancy of $0.33 \mathrm{~m}$ (in planimetry) and $0.67 \mathrm{~m}$ (in altimetry) in the RMSE (Root Mean Square Error) between the two techniques.

Even at present, the possibilities for the use of stereoscopic images from IKONOS and the derivative DEMs are enormous. However, although all of these studies have proposed different uses, all of them contain a variable that determines the validity of these products for each specific purpose, and this variable is the accuracy.

The quality of positional data mapping is one of the most limiting factors for its use. The DEMs are not an exception, and many works [4,9-14] have attempted to best define the configuration of parameters that affect the process of generation and the application of methods that quantify the magnitude of such accuracy.

\section{Variables that affect the generation of a DEM}

The type of sensor influences the DEM generation. The study show a comparison of DEMs obtained with a stereo pair from IKONOS and 
another from EROS-A $[9,10]$. Büyüksalih et al. present and compare the results of an automatic correlation process achieved with highresolution images from QuickBird, IKONOS and OrbView-3. The work [15] compiled the results of over ten generation experiments from several optical and radar satellites. Another study [11], explore the potential of IKONOS imagery for DEM generation and compare the result with a model obtained from LIDAR techniques in the same area, which produced slightly worse results for the generated DEM using satellite imagery $(0.96 \mathrm{~m}$ RMSE compared with $0.50 \mathrm{~m}$ from LIDAR) [16]. Toutin used LIDAR as a source of greater accuracy in assessing the results of eight generation experiences carried out with SPOT-5, IKONOS, QuickBird and EROS-A imagery.

The methodology followed in the process of IKONOS DEMs generation and the results achieved in terms of accuracy both depend on the combination of a multitude of variables and parameters. The type of ground cover and slope are determining factors in the accuracy. Some previous works in which precision area analysis was carried out $[9,11,12]$ agree that the best results are achieved in flat areas and with bare soil surfaces. However, high-reflectivity areas (such as beaches) or areas covered with light-colored buildings, wooded areas and areas of steep slopes are the most problematic for the matching process [16]. Toutin detected the presence of intolerable errors in the process of matching next to $50 \mathrm{~m}$ in highly reflective areas and justified the degraded accuracy in areas of steep slope due to the appearance of shadows. The results of the altimetric accuracy improve significantly (up to a 1-meter difference) in areas with slopes oriented toward the sun compared with results obtained on hillsides that are shown as shaded in the images.

A physical or rigorous method in the mathematical model should provide more accurate results, according to $[12,14,17]$. From a theoretical point of view, it is logical that use of the Canadian Center of Remote Sensing (CCRS) model should provide better results that take into account the physical reality and the geometry of the images in addition to correcting the distortions due to the platform and the sensor itself [7]. This model was developed by Thierry Toutin and Philip Cheng for IKONOS (valid also for EROS and QuickBird). However, Space Imaging warns that when working with incomplete scenes, this method may introduce significant errors in the modeling. More recently, the Rational Functions (RFs) model or empirical model has stirred great interest in the remote-sensing community for civilian uses because certain high-resolution satellites such as IKONOS have begun to distribute images together with the RFs coefficients (also known as RPC files) [18]. The use of the RFs model has drawn considerable interest in the civil remote sensing community because it supplements the lack of information that is not revealed in many cases by the owners of the satellites, in addition to offering a more generalizable method for multiple sensors than the physical model $[18,19]$; its use is recommended when the image has already been geometrically corrected or when it is not possible to work with the entire scene. To obtain a sensor orientation with better accuracy, the RPCs need to be refined with linear equations requesting more accurate GCPs or, more commonly, with 2D polynomial functions [19]. For the latter option, one or two GCPs are used for zero-order polynomial functions (bi-directional shift) and six to ten GCPs for first- and second-order polynomial functions to compute their parameters with a least squares adjustment process [19]. However, refining polynomial coefficients using GCPs needs many control points that cover the entire planimetric and height range of the scene and this can be very difficult to achieve in practice, especially in scenes with distribution restrictions, as coastal areas. Previous works with IKONOS images $[4,12]$ showed that using first- and second-order polynomial functions to refine the IKONOS RPC does not significantly improve the final accuracy when compared to just a bias compensation (zero order). The main reason is because there is no further systematic geometric distortion in the georeferenced IKONOS images, except the relief. Conversely, using higher degree polynomials can also lead to severe extrapolation errors and possible undulations between GCPs.

The GPS is considered to be the capture technique of GCPs unquestionably more employed in this type of work in spite of their limitations as to the difficulty of data collection in wooded areas or areas with high buildings, since it provides an optimal relationship between accuracy and time of data acquisition. The study by Baiocchi et al. includes an example of practical application of the GPS methodology in kinematic mode used for lines of support and control [9].

The influence of the distribution of the GCPs on the results has been analyzed in different studies [20-22], which indicate that the points at the edges of the image are particularly important in obtaining a better fitting. A sufficiently regular grid of points distributed over the entire image achieved good results, although the outside points are more influential in the overall goodness of the adjustment than the inner points [20].

The number of GCPs is a factor that largely depends on the mathematical method used. For the rigorous method, the use of a minimum number of six points is recommended [16]. In the case of the RFs, the coefficients are provided in a text file attached to the images, and the procedure can be performed without the use of any given point, although the addition of certain points greatly enhances the accuracy. Tie points can be added to GCPs and are simply aimed at strengthen the matching process. The points are homologous in the two images and are used to assist the program; therefore, it is not necessary to know their coordinates, but they must be perfectly identifiable in both shots. In high-resolution imagery, the autocorrelation performed by the program is usually sufficiently accurate to eliminate the use of these types of points, although it might be interesting to study their influence.

The number of Independent Check Points (ICPs) used to verify the accuracy of the DEMs is variable, although almost all works use a number between 20 and 30 points for the full scene $\left(121 \mathrm{~km}^{2}\right)$. The exception is the work [11], which uses a much higher number of 111 points for the same surface. The use of specific accuracy standards for quality control would serve to establish common criteria because most of the standards include a minimum set of guidelines related to the number of points to use.

The utility of the previous radiometric processing of the images is a question that is not sufficiently considered. Some studies [4], recommend the use of a low-pass filter to reduce the noise in the images and other filters to enhance the edges, increase the contrast, and improve the correlation between the images, although the effects of application are not analyzed [16]. Toutin indicates that IKONOS images, unlike others (E.g. SPOT), are already distributed with a radiometric correction and that the images taken in the alongtrack mode (in the case of IKONOS) do not require radiometric preprocessing. The ability to collect pairs of images in the same pass and over a short interval of time, allows the IKONOS images to produce more satisfactory results in the matching process than the images taken in cross-track mode because the first case eliminates 
Citation: Gil-Docampo M, Arza-Garcia M (2017) Optimal Parameters for DEM Extraction from IKONOS data: A case Study of a Coastal Area. Geoinfor Geostat: An Overview 5:5.

variations in the solar inclination and atmospheric conditions and possible changes in the morphology of the terrain objects. For this reason, it does not appear than radiometric processing is a key factor for improvement of accuracy in these images.

Despite the commercial availability of stereoscopic products from space-based sensors and the amount of related studies and specific works, even today, great interest remains in assessing the influence of the main variables that affect the DEM generation process, especially as determined by the constraints of the study area. This work verifies the most potentially influential variables with respect to the accuracy of the final DEM, mainly the mathematical model used in the correlation, the number and quality of the applied GCPs.

\section{Materials and Methods}

\section{Image data}

The working area consist of a coastal zone in the northwest of Spain (Figure 1), that presents an altimetric variation of $270 \mathrm{~m}$ with alternating flat and steeply sloped areas and various types of landcover (sandy, urban, bare ground, areas of low vegetation, forested, etc.)

This work employs the panchromatic channel of two images that compose the stereoscopic pair, and their characteristics are shown in Table 1. This example is the "Reference" product with the Ortho Kit and therefore includes the pre-computed coefficients for the RFs method. The overlapping area is $95 \%$ of the scene, and the PCIGeomatics software was used for DEM generation.

\section{GCPs capture and distribution}

The GCP coordinates were obtained from two different methodologies (from 2-D and 3-D contour maps at a 1:5,000 scale from the SITGA (Territorial Information System of Galicia) and from GPS) to analyze the influence of the quality of the GCPs in the final results.

A total of 64 points that are clearly defined in the image and 1:5,000 cartography were measured using GPS with occupation times of approximately 45 seconds. The majority of the GCPs are selected at crossroads and other well-defined geometrical shapes. Postprocessing, using differential correction, made possible to obtain coordinates with approximate accuracies of $\pm 0.3 \mathrm{~m}$ in $\mathrm{X}$ and $\mathrm{Y}$ and $\pm 0.4 \mathrm{~m}$ in $\mathrm{Z}$. As an approximation of the quality of the measured points on the map, we take a conventionally accepted value of $1 / 3$ of equidistance (i.e. approximately $1.67 \mathrm{~m}$ ).

Because the geometry of the work images is not rectangular and the overlapping area of the stereoscopic pair is partially occupied by the sea, it is difficult to establish regular type distributions. Therefore, the standard distributions established based on the previous analyzed studies will need to be adapted to the available geometry; however, the criterion of external point location has been prioritized to the extent possible in the model zone, as reflected in Figure 2.

\section{DEM generation process}

The study variables for analysis are the mathematical model, the number of GCPs and the accuracy by which the generation experiments of the DEMs will be defined, as detailed in Table 2.

The procedure for automatic DEM generation is shown in Figure 3. In our work, the final DEM is achieved when the interpolation and filtering is applied.
In this case, the parameters are defined as the output projection of the DEM or its resolution. We chose to generate the models at the highest possible resolution (i.e., with mesh units similar to the pixel size of the images or $1 \mathrm{~m}$ ) such that this factor has as little impact as possible on the results of positional accuracy [23]

In case of IKONOS, although the values of the parameters are not revealed directly to the user, programs exist that include algorithms and methods that enable modeling (with the assistance of GCPs) of their approximate values and use for DEM generation (the case of the CCRS Model). The values of the pre-computed coefficients that are supplied with the images are also available; therefore, the generation process can be also carried out with the RFs. The vendor's RPCs data and the refined coefficients by a zero-order polynomial adjustment are used in this test, based on previous studies analyzed. The CCRS model requires the introduction of at least six GCPs marked in the two images (Stereo GCPs). The RFs model, however, does not require strictly any GCPs, although addition of certain points is recommended to strengthen the accuracy.

Once the GCPs are entered, the software is able to calculate the ray adjustments for the stereoscopic pair, providing the best possible solution for the location of each image (a process equivalent

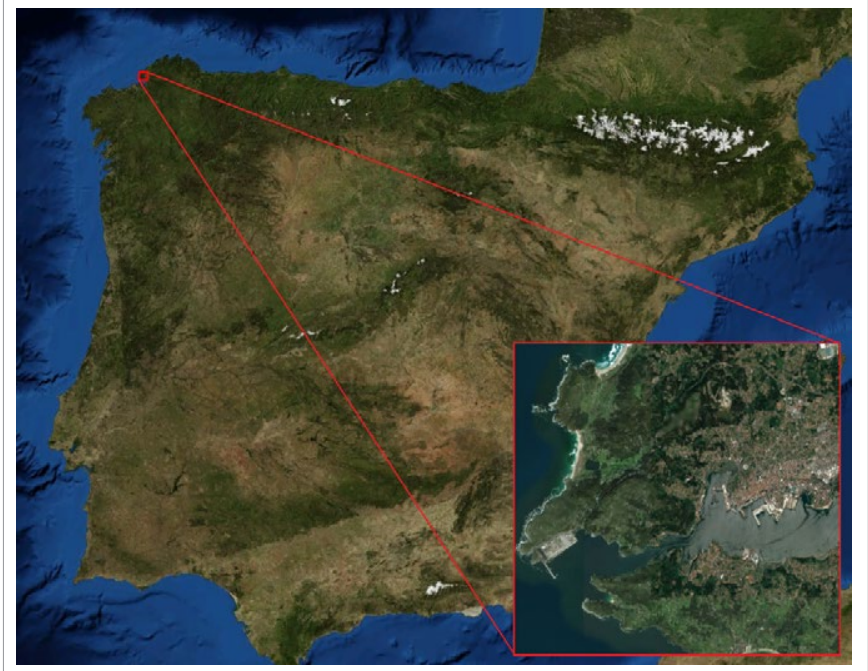

Figure 1: Study area location.

Table 1: Technical specifications of the stereo pair

\begin{tabular}{|l|l|l|l|}
\hline \multirow{2}{*}{ General } & Level & \multicolumn{2}{l|}{ Reference } \\
\hline & Accuracy & $\mathbf{2 5 , 4}$ m CE90 \\
\hline $\begin{array}{l}\text { Interpolation } \\
\text { method }\end{array}$ & \multicolumn{2}{l|}{ Cubic Convolution } \\
\hline & Projection & UTM & \\
\hline & Datum & WGS84 & \\
\hline Acquired nominal GSD & Cross Scan & $0.95 \mathrm{~m}$ & $0.87 \mathrm{~m}$ \\
\hline Nominal collection azimuth & Along Scan & $0.98 \mathrm{~m}$ & $0.88 \mathrm{~m}$ \\
\hline Nominal collection elevation & $38.3508^{\circ}$ & $146.0353^{\circ}$ \\
\hline Sun angle azimuth & & $63.11247^{\circ}$ & $73.37280^{\circ}$ \\
\hline Sun angle elevation & & $156.1555^{\circ}$ & $156.4792^{\circ}$ \\
\hline Number of pixels. & Column & $43.33284^{\circ}$ & $43.40479^{\circ}$ \\
\hline & Row & 12544 & 11920 \\
\hline
\end{tabular}


Citation: Gil-Docampo M, Arza-Garcia M (2017) Optimal Parameters for DEM Extraction from IKONOS data: A case Study of a Coastal Area. Geoinfor Geostat: An Overview 5:5.
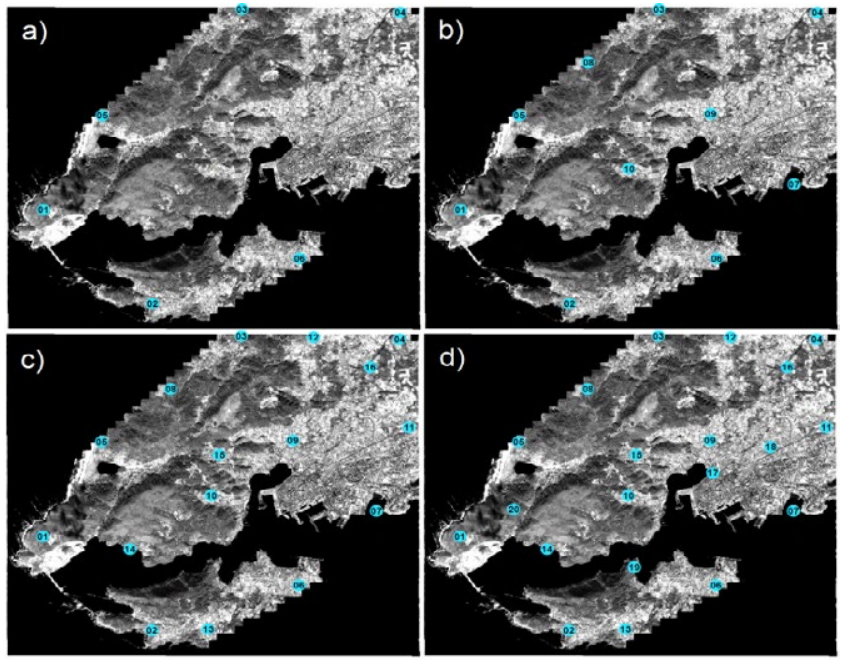

Figure 2: Standard GCP distributions selected for generation testing with a) 6 GCPs, b) 10 GCPs, c) 16 GCPs and d) 20 GCPs.

Table 2: Design of generation trials.

\begin{tabular}{|c|c|c|}
\hline Mathematical model & Number of GCPs & GCP Type \\
\hline \multirow{8}{*}{ Toutin's model (rigorous) } & \multirow{2}{*}{6} & GPS \\
\hline & & Cartography \\
\hline & \multirow{2}{*}{10} & GPS \\
\hline & & Cartography \\
\hline & \multirow{2}{*}{16} & GPS \\
\hline & & Cartography \\
\hline & \multirow{2}{*}{20} & GPS \\
\hline & & Cartography \\
\hline \multirow{9}{*}{$\begin{array}{l}\text { RFs model (with supplied } \\
\text { coefficients) }\end{array}$} & \multicolumn{2}{|l|}{ Without GCPs } \\
\hline & \multirow{2}{*}{6} & GPS \\
\hline & & Cartography \\
\hline & \multirow{2}{*}{10} & GPS \\
\hline & & Cartography \\
\hline & \multirow{2}{*}{16} & GPS \\
\hline & & Cartography \\
\hline & \multirow{2}{*}{20} & GPS \\
\hline & & Cartography \\
\hline
\end{tabular}

to the External Orientation). The approach used to provide this solution involves minimizing the sum of the squares of the errors (Least Squares). As a result of this process, no individual GCP fits perfectly and a few residual values (RMSE) appear that represent the displacements with respect to the calculated positions. Normally, the aim is to achieve residual values of less than one pixel, assuming the capacity to measure the GCPs with as much or more accuracy than the resolution of the images. A high RMSE most likely implies that the DEM accuracy is reduced; however, a high RMSE does not necessarily ensure that the accuracy of the final model is high. The quality of the positional model must be analyzed independently by the ICPs.

Once the adjustment has been carried out, it is possible to proceed to the generation of the pair of normalized images, which are subsequently used to obtain the altimetric information based on the parallax differences. Horizontal parallaxes only exist in the pair of normalized images; the vertical parallaxes are removed in the orientation process; therefore, the process of matching is simplified by assigning all of the homologous points an identical value for the ordinate of the image. In the DEM generation, the parallax differences measured on the normalized images are transformed into altitude differences and referenced to the absolute system. The result of this process is a matrix of pixels whose gray-level values correspond to the middle altitudes of the field in each area occupied by their own pixels.

\section{Results and Discussion}

\section{DEM quality evaluation}

In Figure 4, it is possible to observe the general aspect of the generated model. As shown, the failed matching area of the DEM is mainly the area corresponding to the southwest, in which large gaps caused by poor correlation (related to the presence of clouds and their shadows projected on the ground) render the model incomplete. Figure 5 shows the results of the positional accuracy of the DEM generation experiments in terms of RMSE and LE95.

The maximum levels of positional accuracy were obtained with the rigorous method and 16 GCPs measured using GPS. This combination of variables provides the models with RMSE values equal to nearly one pixel size of the original images $(1.01 \mathrm{~m})$. For this trial, the values of LE95 are less than $2 \mathrm{~m}$.

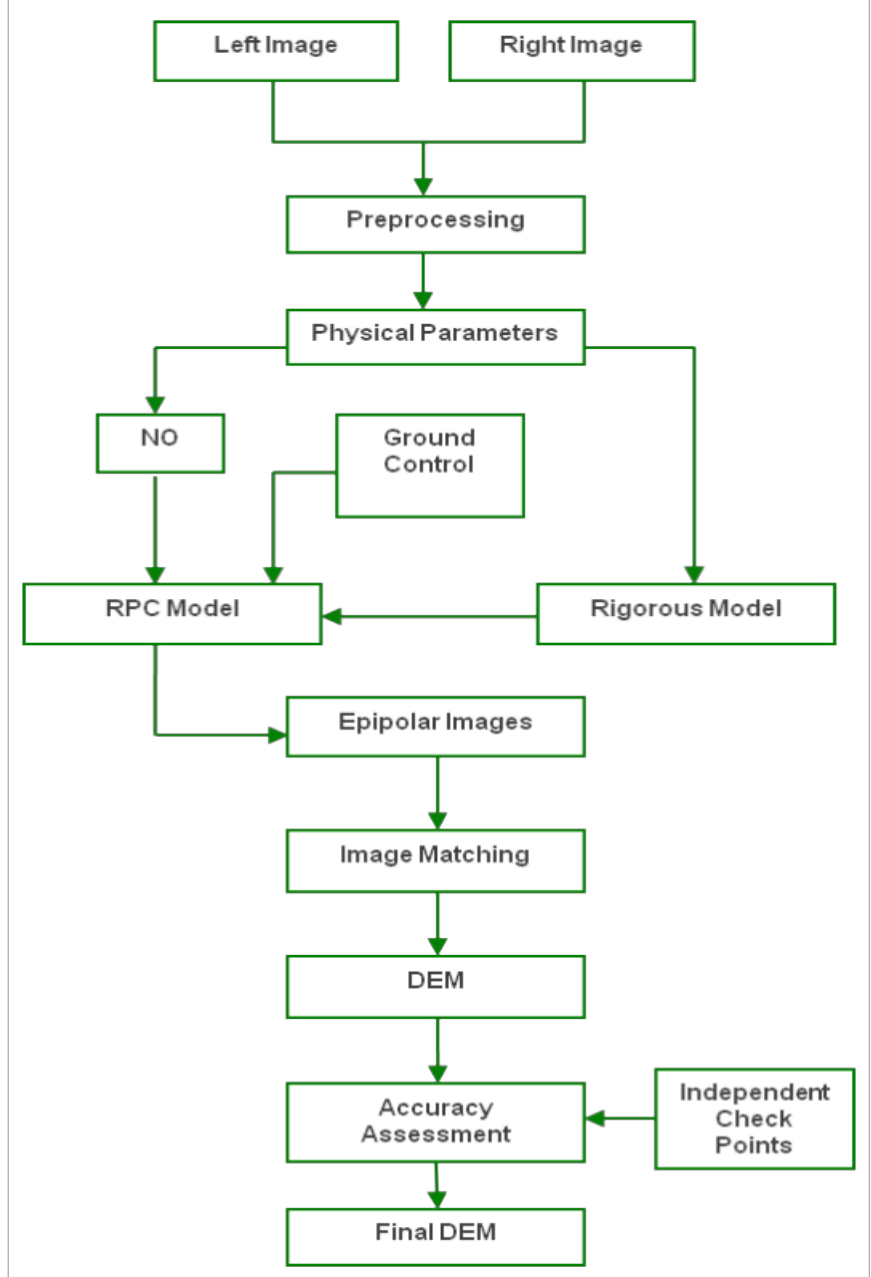

Figure 3: Procedure for automatic DEM extraction [23]. 
Citation: Gil-Docampo M, Arza-García M (2017) Optimal Parameters for DEM Extraction from IKONOS data: A case Study of a Coastal Area. Geoinfor Geostat: An Overview 5:5.

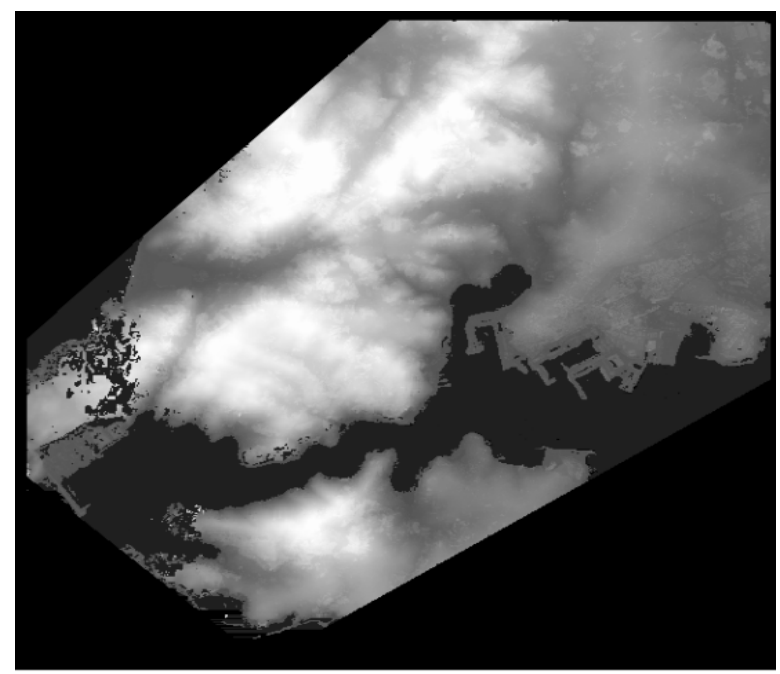

Figure 4: DEM generated from an IKONOS stereo pair.

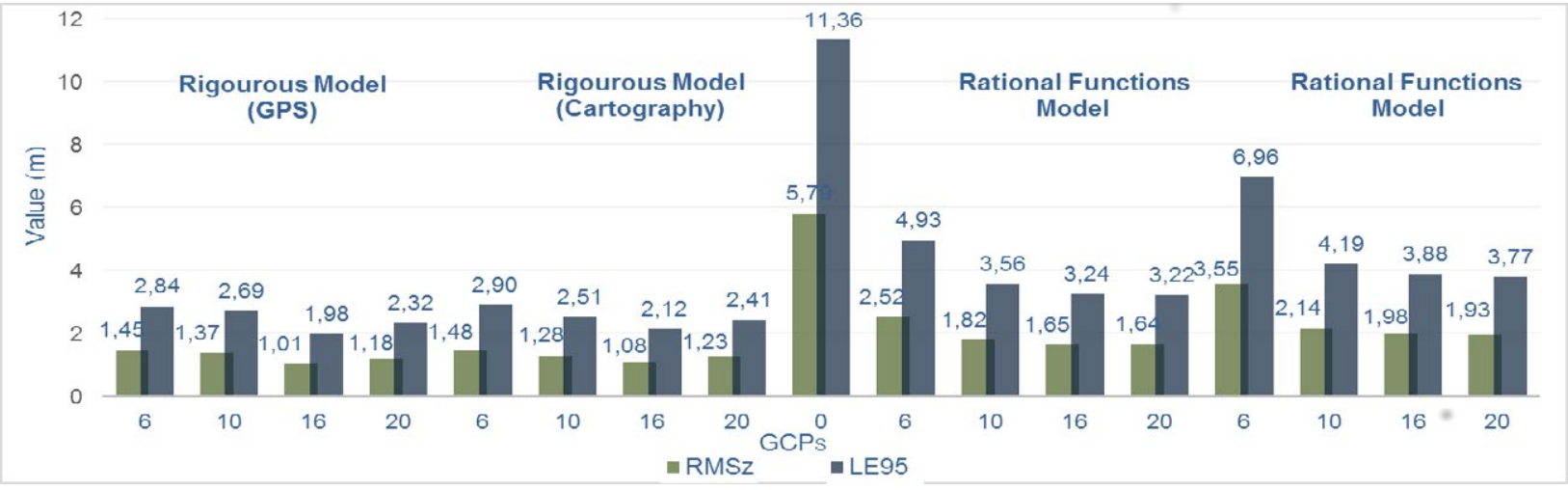

Figure 5: Graph of RMSE and LE95 obtained in each trial generation.

\section{Variables that affect the DEM accuracy}

Mathematical model: According to the Figure 5, we note that the results obtained from comparison of the sample of the 30 ICPs confirms a subset of the underlying assumptions, such as the rigorous method is the most precise mathematical modeling method, with RMSE values less than $1.5 \mathrm{~m}$ in all cases. Moreover, this result is the most important determining factor in the final accuracy because the use of the RFs method does not provide good results, even with the greatest number of points or better-positioned points.

Number of GCPs: The influence of the number of GCPs is evident in the results of RMSE, which improved by approximately $30 \%$ between the trials with 6 and 16 GCPs. In any case, we note that addition of greater than 16 GCPs does not provide improvement and may even produce slightly worse results. In a similar study [12], the rigorous method provided the highest RMSE values with 55 GCPs instead of 12 .

In the RFs method, a clearer trend toward the stabilization of the error from a certain number of GCPs is observed. As in the case of the rigorous method, it does not seem logical to add more than 16 GCPs because the improvement in positional quality is not significant.

It is necessary to comment on the case of DEMs generated without any GCPs as support because, although this approach might have applicability in certain cases, this trial showed results that were significantly worse than any of the other cases. However, the employment of this approach may be feasible for situations in which the availability of GCPs is a conditioning factor and may be interesting in cases in which the requirements for accuracy are not high and the economic and temporal performance is the priority. It was found that the addition of certain GCPs greatly enhanced the accuracy. In fact, with only six points measured using GPS, the RMSE was reduced to less than half.

Positional Quality of GCPs: The accuracy of the GCP source was checked to verify that there were no considerable differences between the use of one source or another, although the GPS provides a vertical accuracy of $\pm 0.4 \mathrm{~m}$ and approximately $1.67 \mathrm{~m}$ for 1:5,000 cartography. Similar results are attributed to the impossibility of marking points in the image with subpixel accuracy, especially in rural areas in which the majority of the GCPs are defined by crossroads or elements that are not well defined [24]. This means that the accuracy in the marking of points, and not the GPS itself, is the source that introduces the greatest error; therefore, the accuracy of the GPS (less than half the size of a pixel) does not necessarily improve the results in an appreciable way. For the cartography, the results are slightly worse than those obtained using GPS; however, the choice will depend on the further implementation of the DEM and the availability of the data or the costs that can be assumed. 
Citation: Gil-Docampo M, Arza-Garcia M (2017) Optimal Parameters for DEM Extraction from IKONOS data: A case Study of a Coastal Area. Geoinfor Geostat: An Overview 5:5.

The physical method appears to be less influenced by this factor than the RFs method. In fact, the results of the rigorous method with different types of support (GPS or cartography) differ rather insignificantly among themselves. In the trial generation experiment with $10 \mathrm{GCPs}$, slightly better results were achieved with points measured on the map, although this result does not have a logical justification beyond the presence of inadvertent errors, and it is not caused by employing GCPs with worse positional accuracy.

\section{Conclusion}

Predictably, the mathematical modeling method that produces DEMs with the highest level of accuracy is clearly the physical or rigorous method. Of the three analyzed variables, the mathematical method has the greatest effect on the final accuracy of the DEM. The use of anywhere between 10 and 16 GCPs provides high levels of accuracy. The stabilization of the error begins with these values and even increases, but the use of a larger number of points is not recommended. The results with GCPs measured from cartography are similar to those measured with GPS, although the estimated altimetry accuracy of the cartography is approximately four times lower. For the RFs method, the differences in the model accuracy that can be expected between the two sources fall in the interval of 0.3-1 m and can be reduced by increasing the number of GCPs. The selection will therefore depend on the subsequent application of the DEM, the availability of data and the costs that can be assumed. However, use of the physical method is more robust in this sense because it does not seem logical to assume the increase in costs and time that justify the use of GPS because the results of the RMSE are quite similar.

In the model, It is possible to achieve RMSE values of $1.01 \mathrm{~m}$, nearly equivalent to the pixel size of the pair of images, using the physical method and 16 GCPs measured with GPS. For this trial, the values of LE95 are less than $2 \mathrm{~m}$, or in other words, $95 \%$ of the points are less than $2 \mathrm{~m}$ from their exact altimetric positions. In terms of accuracy, the results are quite consistent with those of other previous studies. The most notable exceptions are [4], [8], which reached RMSE values of less than a meter with the RFs method; it was not possible in this work to achieve values of less than $1.64 \mathrm{~m}$ with this same method.

Despite the fact that the test scene is partially covered by sea (which determines the distributions of the points), the results obtained are generally no worse than those obtained from previous experience in inland areas. The use of standard distributions, which cover the edges and the inside available zone as homogeneously as possible, can account for this similarity with the results of other works.

\section{Acknowledgment}

This study has been carried out in the framework of the "Financial aid for the consolidation and structure of competitive units of investigation in the universities of the University Galician System (2016-18)" Ref. ED431B 2016/030. This work has been also supported in the same program by Ref. ED341D R2016/023.

\section{References}

1. Wehr A (2009) LiDAR systems and calibration, Topographic Laser Ranging and Scanning: Principles and Processing. Taylor and Francis Group, Florida, USA.

2. Leberl F, Mayr W, Domik G, Kobrick M(1988) Sir-b Stereo-radargrammetry of Australia. Int J Remote Sens 9: 997-1011.

3. Rosen PA, Hensley S, Joughin IR, Li FK, Madsen SN, et al. (2000) Synthetic aperture radar interferometry. Proceedings of the IEEE 88: 333-382.

4. Fraser CS, Baltsavias E, Gruen A (2002) Processing of Ikonos imagery for submetre 3D positioning and building extraction ISPRS Jr Photo Remote Sens 56: 177-194.
5. Krauß T, Lehner M, Reinartz P (2010) Generation of Coarse 3D Models of Urban Areas From High Resolution Stereo Satellite Images. ISPRS Archives, Germany.

6. Aguilar MA, Saldana MDM, Aguilar FJ (2014) Generation and quality assessment of stereo-extracted DSM from geoeye-1 and worldview-2 imagery. IEEE Trans on Geosci Remote Sens 52:1259-1271.

7. Kaichang D, Ruijin M, Rongxing L (2003) Geometric Processing of Ikonos Stereo Imagery for Coastal Mapping Applications. Photogram Engg Remote Sens 69: 873-879.

8. Shaker A, Yan WY, Easa S (2010) Using Stereo Satellite Imagery for Topographic and Transportation Applications: An Accuracy Assessment. GISci Remote Sens 47: 321-337.

9. Baiocchi V, Crespi M, De Vendictis L, Mazzoni A (2005) DSM extraction from IKONOS and EROS A stereo imagery: methodology, accuracy and problems. InProc of EARSeL Workshop 3D Remote Sensing, Portugal.

10. Büyüksalih G, Jacobsen K (2006) Comparison of DEM Generation by Very High Resolution Optical Satellites. $26^{\text {th }}$ EARSeL Symposium on New Developments and Challenges in Remote Sensing, Warsaw, Poland.

11. Poon J, Fraser CS, Chunsun Z, Li Z, Gruen A (2005) Quality assessment of digital surface models generated from IKONOS imagery. Photogrammetric Rec 20: 162-171.

12. Toutin T (2004) Geometric processing of remote sensing images: models, algorithms and methods. Int J Rem sens 25: 1893-1924.

13. Neigh CS, Masek JG, Bourget P, Cook B, Huang C, et al. (2014) Deciphering the precision of stereo IKONOS canopy height models for US forests with G-LiHT airborne LiDAR. Rem Sens 6: 1762-1782.

14. Jeong J, Kim T (2015) Comparison of positioning accuracy of a rigorous sensor model and two rational function models for weak stereo geometry. ISPRS Jr Photogram Rem Sens 108: 172-182.

15. Buyuksalih G, Jacobsen K, Baz I (2008) DEM generation based on optical space images. InEARSeL Workshop Developing Countries. Istanbul, Turkey.

16. Toutin T (2004) Comparison of stereo-extracted DTM from different highresolution sensors: SPOT-5, EROS-A, IKONOS-II, and QuickBird. IEEE trans geosci remote sens 42: 2121-2129.

17. El Sagheer AA, Zaki KM, Gomaa MS, Marrei AM (2017) Rigorous Versus Generalized Sensor Models: Assessment of Different Height Sources for Orthorectification of High-Resolution Satellite Imagery. Surveying and Land Information Science 76: 49-57.

18. Toutin T, Cheng P (2000) Demystification of IKONOS. Earth Obs Magazine 9: 17-21.

19. Poli D,Toutin T (2012) Review of developments in geometric modelling for high resolution satellite pushbroom sensors. Photogram Rec 27: 58-73.

20. Ke LC (2006) Orthorectification Accuracies of VHRS Imagery under the Characteristics of Ground Control Points. International Symposium on Geoinformatics for Spatial Infraestructure Development in Earth and Allied Sciences, Hanoi, Vietnam.

21. Unwin DJ, Drive C (1987) Towards a General Theory of Control Points Distribution Effects in Trend-Surface Models. Comp Geosci 13: 351-355.

22. Mutluoglu O, Yakar M, Yilmaz HM (2015) Investigation of effect of the number of ground control points and distribution on adjustment at WorldView-2 Stereo images. Int Jr App Math, Electro and Comp 3: 37-41.

23. Deilami K, Hashim M (2011) Very High Resolution Optical Satellites for DEM Generation : A Review. Eur Jr Sci Res 49: 542-554.

24. Docampo MG, Corvelle-Rico E, Ortiz-Sanz J (2011) Orthorectification of Quickbird Ortho-ready Imagery: a Case Study Over Montainous Terrain. Survey Review 43: 199-209.

\section{Author Affiliation}

Top

Agroforestry Engineering Department, University of Santiago de Compostela, Higher Polytechnic School, 27002 Lugo, Spain 